\title{
TTR
}

Traduction, terminologie, re?daction

\section{Berman Antoine, La prueba de lo ajeno; Cultura y traducción en la Alemanía Romántica, Traducción de Rosario García López, ed. ULPGC, Universidad de las Palmas de Gran Canaria, 2003.}

\section{Danielle Zaslavsky}

Volume 18, numéro 1, 1er semestre 2005

Traductions et représentations : Parcours dans l'espace hispanique II Translations and Representations: Exploring the Hispanic World II

URI : https://id.erudit.org/iderudit/014378ar

DOI : https://doi.org/10.7202/014378ar

Aller au sommaire du numéro

Éditeur(s)

Association canadienne de traductologie

ISSN

0835-8443 (imprimé)

1708-2188 (numérique)

Découvrir la revue

Citer ce compte rendu

Zaslavsky, D. (2005). Compte rendu de [Berman Antoine, La prueba de lo ajeno; Cultura y traducción en la Alemanía Romántica, Traducción de Rosario García López, ed. ULPGC, Universidad de las Palmas de Gran Canaria, 2003.] TTR, 18(1), 264-266. https://doi.org/10.7202/014378ar d'utilisation que vous pouvez consulter en ligne. 
new in Translation Studies, the present volume constitutes an important contribution to the Hispanic field of Translation Studies, where comparative and evaluative analyses are still presented.

In spite of the multiciplicity of approaches and points of view presented in this volume, it is unfortunate that the concept of literary translation remains reduced to fiction. As in most publications in Translation Studies, works of knowledge are not studied or mentioned here. Perhaps another shortcoming of this volume is that certain articles do not exactly fit within the general theme. It would have been more appropriate to expand on some excellent articles that this book contains, instead of artificially introducing so many. Lastly, the number of typos detracts somewhat from the otherwise professional nature of the work.

\section{References}

DERRIDA, Jacques (1998). Of Grammatology. Translation by Gayatri Chakravorty Spivak, corrected edition. London, The Johns Hopkins University Press.

\section{María Sierra Córdoba Serrano University of Ottawa}

Berman Antoine, La prueba de lo ajeno; Cultura y traducción en la Alemanía Romántica, Traducción de Rosario García López, ed. ULPGC, Universidad de las Palmas de Gran Canaria, 2003.

On ne peut que saluer la traduction en espagnol de l'ouvrage d'Antoine Berman, L'épreuve de l'étranger, traduit en anglais il y a déjà plus de dix ans. ${ }^{23} \mathrm{~S}$ 'il est cité à maintes reprises par un certain nombre de chercheurs dans le monde, c'est au Canada qu'on lui aura fait la place qu’il mérite. $^{24}$

\footnotetext{
${ }^{23}$ Berman Antoine, The Experience of the Foreign: Culture and Translation in Romantic Germany, translated by S. Heywaert, Albany, N.Y, State University of New York, 1992, 250p.

${ }^{24}$ Antoine Berman aujourd'hui, sous la direction d'Alexis Nouss, TTR, 14, 2, 2001.
} 
En effet, Berman a été pour beaucoup d'entre nous - traducteurs et chercheurs en traductologie - le détonateur d'une réflexion qui n’a de cesse aujourd'hui. Si les réflexions livrées par Berman au moment de la publication de son livre n'étaient pas nouvelles pour les intellectuels allemands, elles permettaient au lecteur français intéressé par la problématique de la traduction, de prendre la dimension du rôle joué par le romantisme allemand dans ce domaine. Mais Berman n'en reste pas là. Dans un texte admirable, il actualise les idées forces de la tradition allemande et, en proposant une " analytique de la traduction ", place la traduction au centre de toute réflexion sur le langage et du rapport à l'autre. Ce livre est généreux : si la traduction de la poésie y occupe une place fondamentale, il propose une visée éthique de la traduction qui s'inscrit dans l'ensemble de la problématique culturelle. La récente traduction de Rosario García López vient donc combler une lacune et ne fera qu'accroître la réception de Berman non seulement chez nos étudiants traducteurs, mais également auprès de tous les lecteurs hispanoaméricains qui s'intéressent à la problématique du langage. $^{25}$

Malgré la qualité de cette traduction, quelques critiques s'imposent quant à l'édition proposée par l'Université de las Palmas de Gran Canaria. Rosario García explique dans son introduction que «El mero deseo de acercar a este autor a los lectores de nuestras universidades, me ha llevado a traducir directamente las numerosas citas con las que el autor ilustra su estudio, y no reproducir las publicadas, por una cuestión de tiempo y porque he considerado que lo importante es que el lector se entere inmediatamente de los que dichas citas dicen" (p.10). Elle ajoute également que la seule citation reproduite dans sa version traduite est celle de M. Foucault, étant donnée son extension, la diffusion et la facilité d'accès du texte de Foucault en espagnol. La traductrice, dans un grand souci d'honnêteté, a donc laissé toutes les citations en français - les unes traduites par Berman lui-même, les autres, quand elles existaient, extraites de traductions publiées - et ajouté sa traduction à la suite.

Je me demande si cela était nécessaire. Le résultat donne un livre beaucoup plus volumineux en espagnol qu'en français, alourdi par l'épaisseur d'un papier glacé dont le reflet gêne même parfois la lecture.

\footnotetext{
${ }^{25}$ Malgré l'absence d'une version en espagnol de son texte, Berman a rencontré de nombreux échos en Amérique latine. Voir l'article de Georges Bastin dans le numéro de TTR mentionné ci-dessus.
} 
Par ailleurs, on se trouve face à un texte en abyme puisqu'on ne peut s'empêcher d'observer le travail de la traductrice (généralement de bonne qualité) en procédant à la comparaison avec un original généralement traduit. Cette visibilité du travail de traduction, souligné dans une préface qui s’intitule « Notas de la autora » et signée par « La traductora " est aussi audacieuse que surprenante. Si cette stratégie relève d'une question qui n'est pas nouvelle - quel est le statut du traducteur d'une œuvre littéraire ? - il me semble que la mention du nom de la traductrice sur la couverture et la pertinence de ses notes auraient suffi à lui rendre hommage.

Ceci nous conduit à une autre observation liée à la précédente. L'ouvrage publié par l'Université de las Palmas fait l'effet d'un manuel, de par sa facture même - les titres et les numéros de chapitres soulignés en gris occupent toute une page blanche, - mais également par cette mise en rapport des citations doublement traduites, ce qui je crois n'était pas l'objectif de Berman. Publié au départ dans la collection «Essais » de Gallimard, L'Epreuve de l'Etranger relève d'une réflexion philosophique, dont les retombées pédagogiques sont allées bien au-delà de ce que Berman avait sans doute prévu. La dimension pédagogique de La Prueba de lo Ajeno répond indéniablement à une volonté extrêmement louable, par ailleurs, d'inscrire ce livre au rang des lectures universitaires. Mais si le livre de Berman a occupé les tables de chevet de beaucoup d'entre nous, c'est précisément parce que ce n'était pas un manuel mais un ouvrage riche d'enseignements.

\section{Danielle Zaslavsky El Colegio de México}

